\title{
On the Capacity Region of MANET: Scheduling and Routing Strategy
}

\author{
Michele Garetto, Member, IEEE, Paolo Giaccone, Member, IEEE, and Emilio Leonardi, Member, IEEE
}

\begin{abstract}
We characterize the capacity region of a mobile ad hoc network (in which nodes employ the store-carry-forward communication scheme) and move according to an arbitrary ergodic mobility process. We identify the class of scheduling policies achieving maximum throughput and introduce a joint scheduling and routing formulation that maps the problem into a multicommodity flow over an associated contact graph. Previous capacity results have been derived under the strong assumption that nodes are identical and uniformly visit the entire network area, resulting in a fully connected homogeneous contact graph in which a simple two-hop routing scheme is optimal. Our approach allows extending the analysis to heterogeneous nodes with anisotropic mobility patterns, as typically encountered in realistic mobility traces. In particular, we apply our framework to an experimental network based on vehicular mobility and show that, in scenarios with inhomogeneous contact times, the two-hop routing strategy can significantly be inefficient in terms of throughput and delay.
\end{abstract}

Index Terms-Delay-tolerant networking (DTN), network capacity, routing.

\section{INTRODUCTION}

$\mathbf{I}$ N RECENT years, delay-tolerant networking (DTN) has emerged as a new area of research with many promising applications [3], [4]. DTNs are characterized by intermittent connectivity, network partitioning, long and variable delays, and high error rates. Such performance-challenging conditions can be found in many different environments such as vehicular networks, sparse sensor/actuator networks, in-the-field military or disaster-relief networks, satellite and deep-space interplanetary communications [5], and terrestrial networks serving remote or rural areas. Interesting real-life experiments comprise "pocket-switched networks" based on human mobility [6], networks based on public transportation systems [7]-[9], wildlife tracking [10], and rural kiosks providing Internet access in developing nations [11].

A typical DTN scenario consists of a sparse network of fixed or mobile devices, where most of the time there does not exist a complete path from a source to a destination, or such a path is highly unstable and may soon break. Over time, different links come up and down due to node mobility. This implies that a

Manuscript received March 5, 2008; revised June 4, 2008. First published August 26, 2008; current version published April 22, 2009. This paper was presented in part at the 26th Annual IEEE Conference on Computer Communications, Anchorage, AK, May 6-12, 2007, and in part at the IEEE International Conference on Communications, Glasgow, U.K., June 24-28, 2007. The review of this paper was coordinated by Prof. A. Boukerche.

M. Garetto is with the Dipartimento di Informatica, Università di Torino, 10124 Torino, Italy.

P. Giaccone and E. Leonardi are with the Dipartimento di Elettronica, Politecnico di Torino, 10129 Torino, Italy (e-mail: paolo.giaccone@polito.it).

Digital Object Identifier 10.1109/TVT.2008.2004621 message can be sent over an existing link and be buffered at the receiving node for quite a long time before being forwarded on the next available link toward the destination. While being stored at a mobile node, a message is physically carried to a different location in the network area. This new communication paradigm is usually referred to as store-carry-forward or mobility-assisted routing. This is particularly interesting in the context of vehicular networks, where a fixed communication infrastructure is not available and the cooperation among the vehicles is needed to support the communication.

Node mobility plays a fundamental role not only in providing end-to-end connectivity in the first place but also because it can dramatically increase the overall transport capacity of interference-limited wireless networks, allowing them to scale up to a large number of nodes. Indeed, the wireless networks of static nodes are known to suffer severe per-node throughput decay (on the order of $1 / \sqrt{n}$ ) as the number of nodes $n$ goes to infinity [12]. In contrast, Grossglauser and Tse have first shown in [13] that a simple two-hop relay scheme can keep the pernode throughput constant under an ideal mobility process in which each node independently and uniformly visits the entire network space.

While the work of Grossglauser and Tse suggests that DTNs can indeed scale up to large sizes, and that the two-hop routing strategy is able to fully exploit the transport capacity offered by node mobility, it is unclear whether these results are applicable to the previously mentioned real-life scenarios. One reason is that the analysis in [13] strongly relies on the following assumptions: 1) all nodes are identical; 2) each node uniformly visits the entire network area according to an ergodic mobility process; and 3) the trajectories of different nodes are independent and identically distributed (i.i.d.). Notice that these same assumptions have been maintained in the several papers that, after [13], have analyzed asymptotic delay-capacity tradeoffs, like [14]-[16].

In many practical settings, the above assumptions on node behavior do not hold, in particular the one that the mobility process of each node uniformly covers the entire space over time, making all the nodes basically indistinguishable from each other. This observation is particularly true for vehicular networks, in which mobility is restricted along the roads. In addition, both everyday life experience and campus- or citywide traces containing spatial information (i.e., based on GPS coordinates or radio beacons from base stations and access points) [17]-[20] suggest that a node spends most of the time just in a small portion of the network area, comprising a few frequently visited "significant places" [21], and rarely goes outside this region. Although any two nodes are likely, in the 
long run, to eventually come in contact with each other, the impact of rare contacts on the overall network capacity has to be carefully investigated.

In this paper, we relax the "homogeneous mixing" assumption on node behavior and extend the capacity analysis to heterogeneous nodes with general mobility patterns. First, we provide a general framework within which the capacity analysis of mobile ad hoc networks can be carried out. More specifically, we only require the mobility processes of the nodes to be jointly stationary and ergodic, though possibly correlated (e.g., group movements, which often occur in vehicular mobility), as defined in Section IV. Under this fairly general assumption, in Section V, we formally prove that the class of scheduling policies based only on the instantaneous node position information achieves the maximum network throughput. This means that the capacity region of a mobile network depends on the mobility process only through the joint stationary distribution of the nodes and not on the details about how nodes change their speed and direction over time. Moreover, no gain in terms of throughput can be obtained by scheduling policies using dynamical variables such as instantaneous queue lengths, age of stored information at the nodes, history of node behavior, and past encounters. ${ }^{1}$

Armed with this result, we map the problem of determining the transport capacity of a mobile network into a joint scheduling and routing problem that only requires knowledge of the stationary distribution of nodes over the network area. The capacity region is then obtained by solving a multicommodity flow over the contact graph defined by the scheduling policy and described in Section VI.

We have applied our framework to a specific example of experimental vehicular network, although its validity is more general and applies to any DTN. In the considered scenario, we have observed that the contact graph is almost fully connected; however, the link capacities are highly inhomogeneous, spanning several orders of magnitude. This fact motivated us to investigate the performance of the two-hop routing scheme, originally proposed under the "homogeneous mixing" assumption, in a more realistic case. Our theoretical analysis (presented in Section VII for the case of finite number of nodes and Section VIII for the case of infinite number of nodes) suggests that the two-hop scheme can incur significant throughput losses in the presence of highly asymmetric contact graphs. Therefore, smart routing algorithms discovering links with high available capacity and possibly routing messages over paths of several hops are indeed necessary to exploit the network capacity under a realistic mobility process. In contrast, we emphasize that most of the routing protocols proposed so far for DTN are oblivious of link capacities and aim at discovering minimum-hop paths or minimizing the instantaneous traffic congestion at the nodes (as in [22]).

In Section IX, we compare the performance of different routing strategies in the considered vehicular network in terms of both throughput and delays. The routing algorithms that we

\footnotetext{
${ }^{1}$ Notice that, in this paper, we are only concerned with network throughput and not with message transfer delay. Moreover, we assume an infinite buffer size at the nodes.
}

have compared are the following: 1) the throughput optimal algorithm; 2) the two-hop throughput optimal algorithm; and 3 ) the delay optimal algorithm, which was previously proposed in [23].

Finally, to complete the overview of this paper, in Section II, we discuss related work, and in Section III, we introduce our notation and discuss the main assumptions of this paper. We provide some conclusions in Section X.

We emphasize that our results mainly have a theoretical significance since we ignore several practical issues, such as those related to the implementability of our scheduling/routing schemes, to congestion control, etc. Nevertheless, our results provide useful guidelines for the design of efficient routing/ scheduling strategies.

\section{RELATED WORK}

In the literature related to DTN, routing is the main issue that has been addressed so far. Current proposals to route messages in a DTN strongly depend on the nature of contacts among the nodes (i.e., random or predictable), the amount of information available to them, and the possibility to disseminate or not multiple copies of a message. If the meeting times of nodes are predictable, intelligent routing and forwarding decisions can be made, although optimal schemes (i.e., with complete information) that also account for finite buffers lead to complex linear programming formulations [23]. In [24], the authors assume that the node movements are not only known in advance but can also be controlled to further improve data collection and delivery across the network. More recently, [25] focuses still on periodic movements but exploits multilevel clustering and hierarchical routing based on minimum-delay paths to improve the scalability of the routing algorithms.

When mobility is random, nodes have to communicate during opportunistic unscheduled contacts. Epidemic routing [26] is one of the first proposals to enable message delivery in intermittently connected networks with random mobility. Each node maintains a list of messages whose delivery is pending. Whenever it encounters another node, the two nodes exchange all the messages that they do not have in common. This way, all the messages are eventually disseminated to all nodes, including their destination. Although optimal in terms of minimizing delay, epidemic routing is very wasteful of network resources.

To avoid flooding the entire network, messages can be duplicated in a limited number of copies [27], [28] or probabilistically [29], possibly exploiting history of encounters [30], [31] for a better selection of next hops, combined with various utility functions [32]. Other approaches make use of erasure-coding techniques [33] or network coding [34], [35] to cope with partial data loss and to reduce the routing overhead. Practical routing issues are discussed in [36].

All of the above approaches aim at either minimizing the message transfer delay or maximizing the delivery probability, and they result, in general, in being inefficient in terms of bandwidth exploitation, particularly when employing information duplication/redundancy. In contrast to previous work, in this paper, we study scheduling/routing schemes that aim at maximizing the overall network throughput in the presence of 
heterogeneous nodes with general mobility. To the best of our knowledge, only a few results have appeared in the literature on this subject. In [37], the authors extend the results in [13], proving the asymptotic optimality of the two-hop forwarding scheme in a network where each node independently moves along a randomly chosen great circle on the sphere of the unit surface. The work in [38] studies the maximum rate at which one or more mobile nodes can relay data between a set of static sources and a set of static destinations. Under some simplifying assumptions, they prove that the relay throughput only depends on the stationary distribution of the nodes' position. In particular, they assume that nodes move independent of each other and have the same stationary distribution over the area (not necessarily uniform). In this paper, we extend the analysis to a more general context.

\section{System Assumption AND NotATION}

We consider a mobile ad hoc network composed of $n$ nodes moving according to a general mobility model inside a bidimensional, compact, and convex region $\mathcal{A}$ of area $|\mathcal{A}| . X_{i}(t)$ denotes the position of node $i$ at time $t$, and $\mathbf{X}(t)=\left(X_{1}(t), X_{2}(t), \ldots, X_{n}(t)\right)$ is the vector of node positions. We define with $d_{i j}(t)$ the Euclidean distance between mobile $i$ and mobile $j$ at time $t$, i.e., $d_{i j}(t)=\| X_{i}(t)-$ $X_{j}(t) \|_{2}$.

We assume the node mobility process to be stationary and ergodic, i.e., given any $m$-uple $\left(B_{1}, B_{2}, B_{3}, \ldots, B_{m}\right)$ of Lebesgue measurable subsets of $A$, it results in

$$
\lim _{t \rightarrow \infty} \frac{1}{t} \int_{0}^{t} \mathbb{I}_{\left(\cap_{i} X_{i}(\tau) \in B_{i}\right)} d \tau=E\left[\mathbb{I}_{\left(\cap_{i} X_{i}(t) \in B_{i}\right)}\right], \quad \text { w.p.1 }
$$

where $\mathbb{I}$ represents the logical indicator function. Note that stationarity and ergodicity are rather general assumptions. Most of the mobility models proposed in the literature, such as those described and discussed in [39]-[42], fit within the class considered in this paper.

Node $s$ generates traffic for destination $d$ according to a stationary and ergodic process with average traffic rate $\lambda_{s d}$ bits per second. ${ }^{2}$ We denote by $\Lambda=\left[\lambda_{s d}\right]$ the corresponding $n \times n$ traffic matrix.

We assume that the interference between simultaneous transmissions is described either by the protocol interference model or the physical interference model [12]. According to the protocol interference model, the transmission from node $i$ to node $j$ at time $t$ at rate $r$ is successful only if, for any other simultaneously transmitting node $k$, it holds that

$$
d_{k j}(t)>(1+\Delta) d_{i j}(t)
$$

for some guard factor $\Delta>0$. According to the physical interference model, instead, the transmission from node $i$ to node

\footnotetext{
${ }^{2}$ Defined with $\hat{\lambda}(t, \tau)$, the amount of data generated by a source within the interval $[t, \tau)$, the traffic is said stationary and ergodic with average rate $\lambda$ iff: $E[\hat{\lambda}(t, t+1)]=\lambda$ for any $t>0$ and $\lim _{t \rightarrow \infty} \hat{\lambda}(0, t) / t=\lambda$ w.p.1.
}

$j$ at time $t$ at rate $r$ is successful only if the associated signalto-noise ratio (SNR) is above a given threshold $z$. When the signal power decays with the distance $d$ as $d^{-\alpha}$, the SNR ratio is expressed by

$$
\frac{P_{i} d_{i j}^{-\alpha}(t)}{N_{0}+\sum_{k \neq i} P_{k} d_{k j}^{-\alpha}(t)}
$$

where $P_{k}$ is the power level of transmitter $k$, and $N_{0}$ is the thermal background noise. ${ }^{3}$ Note that according to these interference models, 1) no node can be either the origin or the destination of multiple simultaneous transmissions, and 2) a node cannot simultaneously be the origin and destination of transmissions.

We denote with $E$ the set of all possible transmitter-receiver pairs $(i, j)$ (by construction, it must be $i \neq j$ ). Subsets $\pi$ of $E$ in which nodes appear at most once (either as transmitter or receiver) represent possible transmission configurations, i.e., sets of transmission-receiver pairs $(i, j)$ that may simultaneously be enabled to communicate at time $t$. We denote by $\Pi$ the set of all possible transmission configurations and with $A(t) \subseteq \Pi$ the set of all noninterfering (hence, implementable) transmission configurations at time $t$. A given interference model induces a correspondence between the vector of instantaneous node positions $\mathbf{X}(t)$ and the set of noninterfering transmission configurations $A(t)$. We formalize this concept by introducing the function $\mathcal{I}$ mapping vectors of node positions into sets of noninterfering transmission configurations, i.e., $\mathcal{I}(\mathbf{X}(t))=A(t)$.

Given any set $A$ of implementable transmission configurations, we denote with $\mathcal{I}^{-1}(A)$ the set of node positions $\mathbf{X}$ to which $A$ corresponds through mapping $\mathcal{I}$, i.e., $\mathcal{I}^{-1}(A)=$ $\{\mathbf{X}: \mathcal{I}(\mathbf{X})=A\} .{ }^{4}$ For any $A$, we can univocally determine the probability that $A(t)=A$, i.e., the probability that the configurations in $A$ are implementable at time $t$ as

$$
\begin{aligned}
P(A) & =E\left[\mathbb{I}_{\left.X(t) \in \mathcal{I}^{-1}(A)\right]}\right. \\
& =\lim _{t \rightarrow \infty} \frac{1}{t} \int_{0}^{t} \mathbb{I}_{X(\tau) \in \mathcal{I}^{-1}(A)} d \tau, \quad \text { w.p.1. }
\end{aligned}
$$

Note that the above probability only depends on the joint stationary distribution of the node mobility process.

Finally, we denote with $\left\{T_{n}\right\}$ the sequence of random instants at which the set of implementable transmission configurations changes, i.e., $\lim _{t \uparrow T_{n}} A(t) \neq A\left(T_{n}\right)$.

\section{SCheduling Policy}

The scheduling policy $S$ dynamically selects an implementable transmission configuration $\pi^{S}(t)$ belonging to $A(t)=\mathcal{I}(\mathbf{X}(t))$. In this paper, we restrict our investigation to

\footnotetext{
${ }^{3}$ More complex expressions for the SNR are obtained when considering propagation phenomena such as fading and/or shadowing, the impact of directional antennas, multiple-input-multiple-output (MIMO) systems, etc.

${ }^{4}$ It can be verified that, for any $A \in \Pi, \mathcal{I}^{-1}(A)$ is a convex set of $\mathbb{R}^{2 n}$ for both the protocol and the physical interference models.
} 
stationary and ergodic scheduling policies, i.e., those policies for which

$$
E\left[\mathbb{I}_{(i, j) \in \pi^{S}(t)}\right]=\lim _{t \rightarrow \infty} \frac{1}{t} \int_{0}^{t} \mathbb{I}_{(i, j) \in \pi^{S}(\tau)} d \tau, \quad \text { w.p.1. }
$$

In general, the selection of $\pi^{S}(t)$ may be influenced by several dynamical parameters, including instantaneous queue lengths, age of stored information at nodes, etc. Particularly relevant are those scheduling policies only driven by $\mathbf{X}(t)$. In this paper, we call stateless and memoryless such scheduling policies.

We also introduce the class of simple scheduling policies $\hat{S}$, which is a strict subclass of the stateless and memoryless scheduling policies characterized as follows. At each transition time $T_{n}$, a transmission configuration $\pi \in A\left(T_{n}\right)$ is selected according to a stationary and memoryless (possibly random) rule. The selected transmission configuration is then kept constant in the whole interval $\left[T_{n}, T_{n+1}\right)$. Simple scheduling policies are fully specified by the conditional probabilities $p_{\hat{S}}(\pi, A)$ that the transmission configurations $\pi \in A$ are selected at time $T_{n}$, given that $X\left(T_{n}\right) \in \mathcal{I}^{-1}(A)$

$$
p_{\hat{S}}(\pi, A)=\operatorname{Pr}\left\{\pi^{\hat{S}}\left(T_{n}\right)=\pi \mid X\left(T_{n}\right) \in \mathcal{I}^{-1}(A)\right\}
$$

$\forall A$, and $\pi \in A$. According to scheduling policy $S$, a communication link is established between nodes $i$ and $j$ whose average capacity expressed in bits per second is

$$
\mu_{i j}^{S}=r E\left[\mathbb{I}_{(i, j) \in \pi^{S}(t)}\right]=\lim _{t \rightarrow \infty} \frac{r}{t} \int_{0}^{t} \mathbb{I}_{(i, j) \in \pi^{S}(\tau)} d \tau, \quad \text { w.p.1 }
$$

which, in the case of simple scheduling policies, can be rewritten as

$$
\mu_{i j}^{\hat{S}}=r \sum_{A \in \Pi} \sum_{\pi \in A} \mathbb{I}_{(i, j) \in \pi} p_{\hat{S}}(\pi, A) P(A) .
$$

The fundamental question we would like to answer is "how can we characterize the capacity of the mobile ad hoc network under a scheduling policy $S$ (or $\hat{S}$ )?" To this end, we need to also consider the routing strategy employed to transfer data through the network. The more general and abstract way to define a routing strategy is to specify quantities $f_{i j}^{s d} \in[0,1]$ denoting the average fraction of traffic from node $s$ to node $d$, which is routed through link $(i, j)$, i.e., $j$ follows $i$ as relay node [43] (unless $j=d$ ) and $f_{i i}^{s d}=0$ by construction. The above quantities $f_{i j}^{s d}$ must satisfy the following well-known flow conservation constraints:

$$
\sum_{i} f_{i j}^{s d}-\sum_{k} f_{j k}^{s d}= \begin{cases}1, & \text { for } j=d \\ 0, & \text { for } j \neq d \text { and } j \neq s \\ -1, & \text { for } j=s\end{cases}
$$

A routing strategy specified by a set of $f_{i j}^{s d}$ 's satisfying (9) can be implemented in many ways, for example, by the following hop-by-hop randomized routing algorithm $\mathcal{R}$ : Node $i$ routes data from source $s$ and destined to $d$ by selecting node $j$ as the next hop with probability $f_{i j}^{s d} / \sum_{k} f_{i k}^{s d}$.

\section{Traffic Sustainability and Capacity Region}

In this section, we analyze the performance of a mobile ad hoc network comprising $n$ users, obtaining a precise characterization of its capacity region. ${ }^{5}$ We emphasize that our results are fairly general since only stationarity and ergodicity of traffic and mobility processes are required. We remark that, in our framework, nodes can move in a correlated fashion (like in platoons, groups, etc.), since we do not need to assume independent movements.

Definition 1: We denote the network backlog with $Z(t)$, that is, the amount of traffic (in bits) already generated by sources that have not yet been delivered to destinations at time $t$.

Definition 2: Traffic $\Lambda$ is sustainable if there exists a scheduling policy $S$ and a routing strategy $\mathcal{R}$ such that $\lim \sup _{t \rightarrow \infty} Z(t) / t=0$ w.p. 1 .

Definition 3: Traffic $\Lambda$ is strongly sustainable if there exists a simple scheduling policy $\hat{S}$ and a routing strategy $\mathcal{R}$ such that $\lim \sup _{t \rightarrow \infty} Z(t) / t=0$ w.p.1.

We are now in a position to state our first result.

Theorem 1: A mobile ad hoc network sustains a traffic $\Lambda$ if a scheduling policy $S$ and a routing strategy $\mathcal{R}$ can be found such that

$$
\sum_{s d} \lambda_{s d} f_{i j}^{s d} \leq \mu_{i j}^{S} \quad \forall i, j
$$

Moreover, if a simple scheduling policy $\hat{S}$ and a routing strategy $\mathcal{R}$ can be found such that

$$
\sum_{s d} \lambda_{s d} f_{i j}^{s d} \leq \mu_{i j}^{\hat{S}} \quad \forall i, j
$$

the mobile ad hoc network strongly sustains traffic $\Lambda$.

Proof: The system dynamics can be described by a network of queues representing the evolution of the backlog at different nodes. We suppose that every node $i$ is equipped with $n-1$ separate transmission queues, each one storing data to be routed through a different node $j$. Upon reception, new data are immediately routed according to policy $\mathcal{R}$ and enqueued in the transmission queue associated to the next hop. Transmission queues are served at fixed rate $r$ according to a first come first served service policy during the periods of activity of the corresponding link $(i, j)$. Note that, by construction, the average service rate in bits per second of the transmission queue of link $(i, j)$ is $\mu_{i j}^{S}$. The network of queues describing the system falls in the class of generalized Kelly networks, which are stable under the condition that no queues are overloaded [44]. Being, by construction, the load at the queue of link $(i, j)$ equal to $\sum_{s d} \lambda_{s d} f_{i j}^{s d} / \mu_{i j}^{S} \leq 1$, the assert immediately follows. ${ }^{6}$

As a corollary, we get a strict characterization of the traffic matrices that are strongly sustainable.

\footnotetext{
${ }^{5}$ We only consider the net traffic (goodput), neglecting the effect of the signaling traffic necessary to implement the routing and scheduling algorithms.

${ }^{6}$ When $\sum_{s d} \lambda_{s d} f_{i j}^{s d} / \mu_{i j}^{S}=1$, the concept of stability is weak.
} 


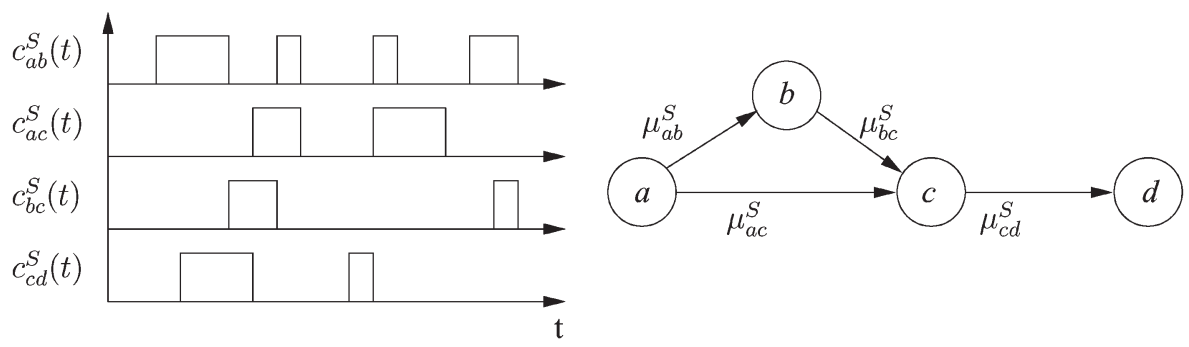

Fig. 1. Contact graph construction. (Left) Temporal sequence of contacts among node pairs. (Right) Corresponding capacitated contact graph.

Proposition 1: A traffic matrix $\Lambda=\left[\lambda_{s d}\right]$ is strongly sustainable iff a set of $f_{i j}^{s d} \in[0,1] \forall i, j, s, d$ and $p(\pi, A) \in[0,1] \forall A$, $\forall \pi \in A$ can be found satisfying the following equations:

$$
\begin{cases}f_{i j}^{s d} \text { satisfies }(9) & \forall i, j, s, d \\ \sum_{\pi \in A} p(\pi, A)=1 & \forall A \in \Pi \\ \sum_{s d} \lambda_{s d} f_{i j}^{s d} \leq r \sum_{A \in \Pi} \sum_{\pi \in A} \mathbb{I}_{(i, j) \in \pi} p(\pi, A) P(A) & \forall i, j .\end{cases}
$$

Definition 4: The capacity region of the mobile ad hoc network is the set of all sustainable traffic matrices.

Definition 5: The restricted capacity region of the mobile ad hoc network is the set of all strongly sustainable traffic matrices.

Note that the restricted capacity region, by construction, depends on node mobility only via the joint stationary distribution of nodes. We can now state the following fundamental result.

Theorem 2: If the traffic matrix $\Lambda$ is sustainable, then it is also strongly sustainable.

Proof: Let $S$ be the stationary and ergodic scheduling policy that sustains $\Lambda$. Define for every configuration $A$ and every $\pi \in A$

$$
\begin{aligned}
q_{S}(\pi, A) & =\operatorname{Pr}\left\{\pi^{S}(t)=\pi \mid X(t) \in \mathcal{I}^{-1}(A)\right\} \\
& =\lim _{t \rightarrow \infty} \frac{1}{t} \int_{0}^{t} \mathbb{I}_{\pi^{S}(\tau)=\pi \mid X(\tau) \in \mathcal{I}^{-1}(A)} d \tau, \quad \text { w.p.1. }
\end{aligned}
$$

Due to the ergodicity of the mobility process and scheduling policy, the above quantities are well defined. It is immediate to verify that $\sum_{\pi \in A} q_{S}(\pi, A)=1$. Thus, considering a stationary simple scheduling policy $\hat{S}$ such that $p_{\hat{S}}(\pi, A)=q_{S}(\pi, A)$, it follows by construction that $\mu_{i j}^{S}=\mu_{i j}^{\hat{S}} \forall i, j$.

The previous result has three significant implications. 1) The class of simple policies achieves maximum throughput, i.e., no gain in terms of throughput can be obtained by adopting complex scheduling policies that select transmission configurations by considering dynamical variables such as instantaneous queue lengths, age of stored information at the nodes, etc., provided that the capacities associated to the communication links are known in advance. 2) A tight characterization of the capacity region is provided by Proposition 1. 3) The capacity region depends on the mobility process only through the joint stationary distributions of nodes. This result extends and generalizes recent findings in [38].

Corollary 1: The capacity region of an ad hoc wireless network with mobile nodes is convex.
Proof: Let $\Lambda_{1}$ and $\Lambda_{2}$ be two sustainable traffic matrices. Let $\hat{S}_{1}$ and $\hat{S}_{2}$ be two simple scheduling policies that sustain $\boldsymbol{\Lambda}_{1}$ and $\boldsymbol{\Lambda}_{2}$, respectively. Any traffic pattern $\boldsymbol{\Lambda}=$ $\alpha \boldsymbol{\Lambda}_{1}+(1-\alpha) \boldsymbol{\Lambda}_{2}$ with $0 \leq \alpha \leq 1$ is sustainable by the simple policy $\hat{S}$ defined according to $p_{\hat{S}}(\pi, A)=\alpha p_{\hat{S}_{1}}(\pi, A)+(1-$ $\alpha) p_{\hat{S}_{2}}(\pi, A) \forall A, \pi \in A$. Note that $\hat{S}$ (at time $t$ ) with probability $\alpha$ emulates $\hat{S}_{1}$ and with probability $(1-\alpha)$ emulates $\hat{S}_{2}$.

\section{Contact Graph: Throughrut and Routing}

To understand the relationship between the scheduling policy and the routing strategy, we first need to characterize which traffic patterns are sustainable by employing an assigned scheduling policy $S$. Observe that the capacities ${ }^{7} \mu_{i j}$ associated to the communication links are univocally determined once the scheduling policy $S$ has been selected. Thus, a (capacitated) graph $G(\mathcal{V}, \mathcal{E})$ whose vertices correspond to network nodes and whose capacitated edges correspond to communication links fully characterizes the mobile ad hoc network adopting $S$. In the following, we refer to $G(\mathcal{V}, \mathcal{E})$ with the term contact graph. Therefore, the routing problem through the mobile ad hoc network adopting $S$ can be formalized in terms of a multicommodity flow problem on the contact graph. Fig. 1 shows an example of contact graph construction. For simplicity, in the figure, we have defined $c_{i j}^{S}(t)=\mathbb{I}_{(i, j) \in \pi^{S}(t)}$, whereas the service rates are evaluated according to (7), i.e., by summing (or averaging) the capacity of all the transmission opportunities between the corresponding nodes.

Proposition 2: A traffic matrix $\boldsymbol{\Lambda}=\left[\lambda_{s d}\right]$ can be sustained by employing a policy $S$ iff the multicommodity flow problem defined by (9) and (10), where the communication link capacities are determined by $S$, admits a feasible solution. In such a case, the set of variables $f_{i j}^{s d}$ univocally defines the routing strategy $\mathcal{R}$.

The sustainable region achievable by the scheduling policy $S$ (i.e., the set of $\boldsymbol{\Lambda}=\left[\lambda_{s d}\right]$ that can be sustained employing $S$ ) can be related to the capacities associated with cuts of the contact graph as follows.

Proposition 3: Traffic $\boldsymbol{\Lambda}=\left[\lambda_{s d}\right]$ is sustainable by policy $S$ only if, for any partition $(D, \bar{D})$ of the nodes, it results in

$$
\sum_{s \in \bar{D}} \sum_{d \in D} \lambda_{s d} \leq \sum_{s \in \bar{D}} \sum_{d \in D} \mu_{s d}
$$

\footnotetext{
${ }^{7}$ To simplify the notation, we omit, in this section, the explicit dependency from the scheduling policy $S$.
} 
Consider a network adopting a scheduling policy $S$ and a routing strategy $\mathcal{R}$ under a sustainable traffic pattern $\Lambda$. Let $\nu$ be the network throughput equal by definition to the offered network load $\nu=\sum_{s d} \lambda_{s d}$, and let $|\pi(t)|$ be the size of $\pi(t)$ (i.e., the number of parallel transmissions enabled by $S$ at time $t$ ), which results in

$$
E[|\pi(t)|]=E\left[\sum_{i j} \mathbb{I}_{(i, j) \in \pi(t)}\right]=\sum_{i j} \frac{\mu_{i j}}{r} .
$$

Letting $C$ be the average aggregate transmission rate over all of the links, by construction, we have

$$
C=\sum_{s d} \lambda_{s d} \sum_{i j} f_{i j}^{s d} \leq r E[|\pi(t)|] .
$$

The ratio $h_{\text {ave }}=C / \nu$ represents the average number of times that data are transmitted in the network; thus, $h_{\text {ave }}$ is the average length of the paths followed by information flows, expressed in number of hops. The following relationship is of immediate verification: $\left.\nu h_{\text {ave }} \leq r E[\mid \pi(t)) \mid\right]$.

In general, to efficiently exploit the network bandwidth, the routing strategy should minimize $h_{\text {ave }}$. This consideration justifies the fact that shortest-path routing approaches have widely been used in several application contexts related to computer communications. In the context of mobile ad hoc networks, the two-hop routing strategy proposed in [13] has gained wide popularity. According to this strategy, data are delivered from source $s$ to destination $d$ either through the direct communication link or through routes $s \rightarrow k \rightarrow d$ using every other node $k$ of the network as relay. Although the twohop routing strategy is appealing because of its simplicity, in general, it does not allow optimally exploiting the network bandwidth, possibly causing a reduction of the sustainability region achievable by the scheduling policy, as we show in the following section.

\section{Performance of The Two-Hop ROUTING STRATEGY}

Necessary and sufficient conditions for traffic $\Lambda$ to be sustainable under a two-hop routing strategy are provided by the following statement.

Proposition 4: A traffic pattern $\Lambda=\left[\lambda_{s d}\right]$ is sustainable by the two-hop routing scheme if the multicommodity flow problem (on the contact graph defined by (9) and (10) with the extra constraint $f_{i j}^{s d}=0$ when both $i \neq s$ and $j \neq d$ ) admits a feasible solution.

The set of all sustainable traffic matrices under the two-hop routing strategy represents the two-hop capacity region of the mobile ad hoc network. In the following, we are interested in better understanding the relationship between the capacity region and the two-hop capacity region on realistic contact graphs associated with mobile ad hoc networks.

To simplify the discussion, in analogy to what has been done in previous works [12], [13], [37], we assume that every node in the network is the source and destination of a single information flow (i.e., $\Lambda$ contains only one nonnull element per row and per column). In the following, we refer to the above class of traffic patterns with the term "permutation traffic patterns."

Of course, the performance of the two-hop routing strategy can arbitrarily be bad on a general contact graph. For example, considering graphs with maximum nodal degree $d$, the average distance between nodes is (using standard Landau asymptotic notation) $\Omega\left(\log _{d}(n)\right)$. Thus, for sufficiently large $n$, if traffic is exchanged only between node pairs whose distance on the contact graph is greater than two, the throughput sustained by the two-hop strategy turns out to be equal to zero. As we will see in the next section, contact graphs associated to mobile ad hoc networks are typically strongly connected [i.e., $d=$ $\Theta(n)]$ and often fully connected (all edges exist). In the latter cases, the contact graph diameter is expected to be very short. On this regard, we recall that condition $d=\Theta(n)$ has been proved to be sufficient to asymptotically guarantee a diameter equal to 2 in several families of random graphs, ${ }^{8}$ such as Erdõs-Rényi graphs and graphs with a regular degree [45]. Nevertheless, we will show that, even when either $d=\Theta(n)$ or $d=n-1$, the two-hop routing may strongly be inefficient provided that the capacities associated to the edges of the contact graph are highly unbalanced.

Under a permutation traffic pattern, let us focus on a particular traffic relation $(s, d)$ and consider all paths from node $s$ to node $d$ followed by data according to a two-hop routing strategy. The aggregate maximum transmission capacity obtainable by flow $s \rightarrow d$ on all parallel one-hop and two-hop paths of the graph is given by

$$
\mu_{s d}^{2 h}=\mu_{s d}+\sum_{k \neq s, d} \min \left(\mu_{s k}, \mu_{k d}\right) .
$$

Moreover, the direct link from $s$ to $d$ is exclusively used by flow $(s, d)$. Link $(s, k)$ must instead be shared by flow $s \rightarrow d$ and the flow generated by $s^{\prime} \neq s, k$ and directed to $k$, whereas link $(k, d)$ is shared by flow $s \rightarrow d$ and the flow generated by $k$ and destined to $d^{\prime} \neq k, d$. Hence, the capacity of each edge can be shared by at most two flows.

Sufficient conditions for traffic sustainability can easily be derived under the two-hop routing strategy assuming that the bandwidth of links is evenly partitioned among the flows.

Proposition 5: Under the two-hop routing strategy, a permutation traffic pattern is guaranteed to be sustainable if, for every source and destination pair $(s, d)$, the following constraint is met:

$$
\lambda_{s d} \leq \mu_{s d}+\frac{1}{2} \sum_{k \neq s, d} \min \left(\mu_{s k}, \mu_{k d}\right) .
$$

It is interesting to understand under which conditions the two-hop routing strategy is guaranteed to efficiently exploit the system bandwidth. The following theorem provides an answer to this question.

Theorem 3: If, for every pair of nodes $(s, d), \mu_{s d}^{2 h}$ is equal to the minimum-cut capacity between $(s, d)$, then the two-hop routing scheme is $1 / 2$ efficient in terms of throughput, i.e., if

\footnotetext{
${ }^{8}$ The above property holds with a probability that tends to 1 when $n \rightarrow \infty$.
} 
$\Lambda=\left[\lambda_{s d}\right]$ cannot be sustained by the two-hop routing strategy, no other routing strategy can sustain traffic $2 \Lambda=\left[2 \lambda_{s d}\right]$.

We remind that, given any partition of graph vertices $(S, D)$ with $s \in S$ and $d \in D$, the capacity $\mu_{(S, D)}$ associated to the cut $(S, D)$ is defined as $\mu_{(S, D)}=\sum_{i \in S} \sum_{j \in D} \mu_{i j}$. The mincut capacity $\hat{\mu}_{s d}$ between $(s, d)$ is defined as $\min _{(S, D)} \mu_{(S, D)}$.

Proof: Consider any source-destination pair $(s, d)$. Under a general routing strategy, the amount of traffic $\lambda_{s d}$ that can be sustained by the network cannot exceed the min-cut between $s$ and $d$ on the contact graph. On the other side, the twohop routing strategy sustains, between $s$ and $d$, a traffic $\lambda_{s d} \geq$ $(1 / 2) \mu_{s d}^{2 h}$ according to Proposition 5. The assert immediately follows since $\mu_{s d}^{2 h}=\hat{\mu}_{s d}$ for all $s$ and $d$.

Since $\sum_{k} \mu_{s k}$ and $\sum_{k} \mu_{k d}$ are two cut capacities, the condition

$$
\mu_{s d}^{2 h}=\min \left(\sum_{k \neq d} \mu_{s k}, \sum_{k \neq s} \mu_{k d}\right)
$$

is sufficient to guarantee that at least half of the optimal throughput is delivered by the two-hop routing strategy under any permutation traffic pattern.

Note that, when nodes uniformly move over the network area $\mathcal{A}$, by symmetry, all link capacities $\mu_{i j}$ are equal; thus, $\mu_{s d}^{2 h}=$ $\hat{\mu}_{s d}$ for all $s$ and $d$. Furthermore, in this specific case, the twohop strategy can easily be shown to be 1 efficient under uniform permutation traffic patterns (i.e., when all nodes generate the same amount of traffic). In this case, indeed, the adoption of the two-hop routing scheme permits to completely saturate the capacity of every link while minimizing the expected number of hops. For a general contact graph $G(\mathcal{V}, \mathcal{E})$, define

$$
\eta^{2 h}=\min _{s d} \frac{\mu_{s d}^{2 h}}{\hat{\mu}_{s d}} .
$$

The statement of Theorem 3 can be generalized to the case $\eta^{2 h}<1$.

Theorem 4: Provided that $\eta^{2 h}=\alpha<1$, the two-hop scheme is $\alpha / 2$-efficient in terms of throughput, i.e., if $\Lambda=\left[\lambda_{s d}\right]$ cannot be sustained by the two-hop scheme, no other routing strategy can sustain traffic $(2 / \alpha) \Lambda=\left[(2 / \alpha) \lambda_{s d}\right]$.

Furthermore, there exists a permutation traffic pattern $\Lambda$ that is sustainable by a properly defined routing scheme and such that $(\alpha+\epsilon) \Lambda$, for any $\epsilon>0$, is not sustainable by the two-hop strategy.

Proof: The first statement can exactly be proved using the same arguments of Theorem 3 .

For what concerns the second statement, consider the source-destination pair $(s, d)$ in correspondence to which $\mu_{s d}^{2 h} / \hat{\mu}_{s d}=\alpha$. If only $(s, d)$ exchanges traffic in the network, the maximum amount of traffic that can be sustained in the network by an arbitrary routing strategy equals the min-cut $\hat{\mu}_{s d}$. On the other hand, according to the two-hop scheme, the maximum amount of traffic that can be sustained cannot exceed $\mu_{s d}^{2 h}$.

In conclusion, if $\eta^{2 h}=\alpha$, we should be prepared to get a throughput reduction of order $2 / \alpha$ in case the two-hop routing strategy is adopted.

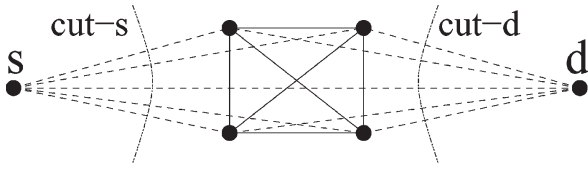

Fig. 2. Contact graph for $n=6$.

\section{Some Asymptotic Considerations on THE THROUGHPUT LOSS OF THE TWO-HOP ROUTING STRATEGY}

In this section, we focus on fully connected contact graphs with inhomogeneous capacities, such as those resulting from experimental traces. We assume that edge capacities $\mu_{i j}$ are i.i.d. random variables with assigned distribution. In this case, we are able to analytically show that, for $n \rightarrow \infty$, the two-hop routing strategy can strongly be inefficient, and that the throughput loss depends on the particular edge capacity distribution.

Essentially, we need to evaluate the parameter $\eta^{2 h}$ introduced in (20). Due to the symmetry of the contact graph, we consider a randomly chosen source-destination pair $(s, d)$ and estimate the ratio between the aggregate capacity of two-hop paths and the minimum capacity among all cuts between $s$ and $d$ as $n$ grows large. In Fig. 2, the contact graph is represented for $n=6$.

As $n$ increases, to keep finite the capacities of the cuts to be evaluated, edge capacities are scaled by $n$, i.e., their distribution is given by $f_{\mu}^{n}(x)=n g(n x)$, where $g(x)$ is an assigned distribution function with average $\hat{\mu}=\int x g(x) d x$. As a consequence, the average capacity of each edge is $E\left[\mu_{i j}\right]=\hat{\mu} / n$. Note that the scaling effect of edge capacities is intrinsically obtained when contact capacities are computed according to (7) (see [13]). Given two independent random variables $X$ and $Y$ distributed according to $g(x)$, we have $\mu_{\min }=E[\min (X, Y)]$.

Observe in Fig. 2 that the cut corresponding to node partition $(S, D)$ with $|S|=k$ and $D=n-k$ (where $k=1, \ldots, n-1)$ contains $k(n-k)$ edges; thus, the cut around the source ("cut-s") (i.e., the cut for which $S=\{s\}$ ) and the cut around the destination ("cut-d") (i.e., $D=\{d\}$ ) are minimal in terms of number of edges traversing them $(n-1)$. This suggests that they are also minimal in terms of capacity.

Indeed, under mild assumptions, standard concentration arguments allow confirming this intuition for large $n$. In particular, we can claim that with high probability (w.h.p.) (i.e., with probability that tends to 1 when $n \rightarrow \infty$ ) the minimumcapacity cut corresponds to either "cut-s" or "cut-d." Hence, the maximum achievable flow from $s$ to $d$ corresponds to the minimum between cut-s and cut-d, i.e.,

$$
\hat{\lambda}_{s d}=\min \left(\sum_{k \neq s} \mu_{s k}, \sum_{k \neq d} \mu_{k d}\right) \rightarrow \hat{\mu}, \quad \text { w.h.p. }
$$

when $n \rightarrow \infty$. If we allow only two-hop routing, the maximum achievable throughput $\lambda_{s d}^{2 h}$ is given by the aggregate capacity 

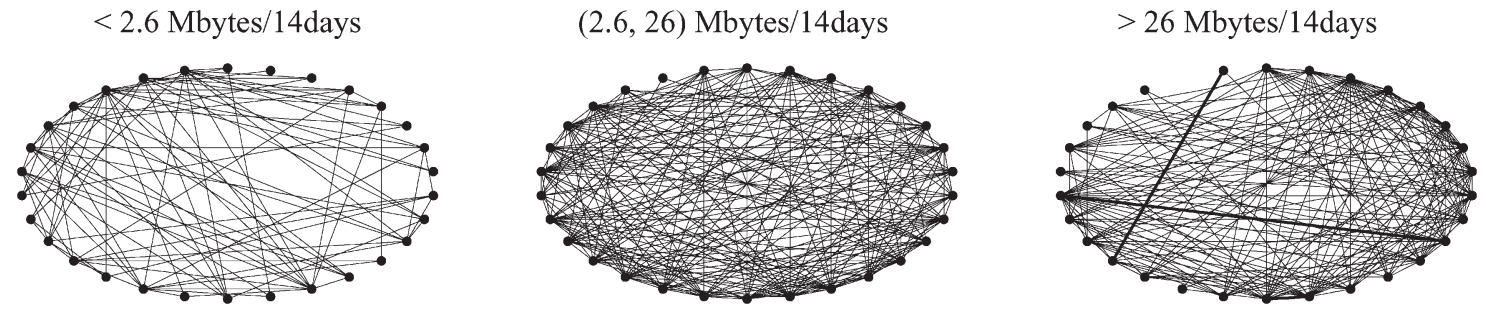

Fig. 3. Contact graphs of Umass buses divided in different classes of edge capacities. Larger capacity edges 3 and 4 contribute $85 \%$ of the overall transport capacity.

TABLE I

COMPARISON OF THE METRICS OPTIMIZED BY DifFERENT Routing Policies

\begin{tabular}{|l|c|c|c|}
\hline $\begin{array}{l}\text { Routing } \\
\text { policy }\end{array}$ & $\begin{array}{c}\text { Maximum } \\
\text { throughput }\end{array}$ & $\begin{array}{c}\text { Minimum } \\
\text { delay }\end{array}$ & $\begin{array}{c}\text { Number } \\
\text { of hops }\end{array}$ \\
\hline TH-OPT & yes & no & any \\
TH-2H-OPT & yes & no & $\leq 2$ \\
DL-OPT [23] & no & yes & any \\
random 2-hop relay [13] & only asympt. & no & 2 \\
\hline
\end{tabular}

on all $n-1$ parallel one-hop and two-hop paths between $s$ and d, i.e.,

$$
\lambda_{s d}^{2 h}=\mu_{s d}+\sum_{k \neq s, d} \min \left\{\mu_{s k}, \mu_{k d}\right\} \rightarrow \mu_{\min } \quad \text { w.h.p. }
$$

when $n \rightarrow \infty$. Hence, asymptotically for $n \rightarrow \infty, \eta^{2 h}$ is given by

$$
\eta^{2 h}=\min _{s d} \frac{\lambda_{s d}^{2 h}}{\hat{\lambda}_{s d}}=\frac{\mu_{\min }}{\hat{\mu}} \quad \text { w.h.p. }
$$

It is interesting to compute $\mu_{\min } / \hat{\mu}$ for specific distributions of edge capacities. We consider two cases, which are both simple to analytically evaluate.

1) $g(x)$ is an exponential distribution with mean $a$ (i.e., $g(x)=e^{-x / a} / a$, for $\left.x \geq 0\right)$. Then, $\min \left\{\mu_{s k}, \mu_{k d}\right\}$ is still exponential with mean $a / 2: \eta^{2 h} \leq 1 / 2$.

2) $g(x)$ is a Pareto distribution with parameters $a$ and $b$ (i.e., $g(x)=b a^{b} / x^{b+1}$, for $x \geq a$ and $b>1$ ) having mean $a b /(b-1)$. Then, $\min \left\{\mu_{s k}, \mu_{k d}\right\}$ is still Pareto with parameters $a$ and $2 b: \eta^{2 h} \leq 2(b-1) /(2 b-1)$. Hence, $\eta^{2 h}$ is upper bounded by an increasing function starting from 0 for $b \rightarrow 1$ and growing to 1 only for $b \rightarrow \infty$. Note that $b \in(1,2]$ corresponds to capacities with finite mean but infinite variance.

Hence, for exponentially distributed capacities, under a generic permutation traffic pattern, we can expect the two-hop routing to be slightly inefficient, leading to throughput loss within a factor of 4 (by Theorem 4). For Pareto-distributed capacities, instead, two-hop routing can be very inefficient, leading to unbounded throughput loss when $b \rightarrow 1$.

In conclusion, in case of inhomogeneous edge capacities, the paths of several hops are needed to efficiently exploit the system bandwidth, even when the associated contact graph is fully connected. This is particularly true when the distribution of link capacities exhibits a long tail, as typically found in experimental traces.
TABLE II

Performance Achievable Under Single-Flow Scenario, Averaged Among All the Possible Flows, for Umass Buses

\begin{tabular}{|c|c|c|}
\hline $\begin{array}{c}\text { Routing } \\
\text { algorithm }\end{array}$ & $\begin{array}{c}\text { Average capacity } \\
\text { per flow }\end{array}$ & $\begin{array}{c}\text { Average } \\
\text { delay }\end{array}$ \\
\hline TH-OPT & 299 Mbytes/14days & 105.7 hours \\
TH-2H-OPT & 134 Mbytes/14days & 52.6 hours \\
DL-OPT & 216 Mbytes/14days & 30.4 hours \\
\hline
\end{tabular}

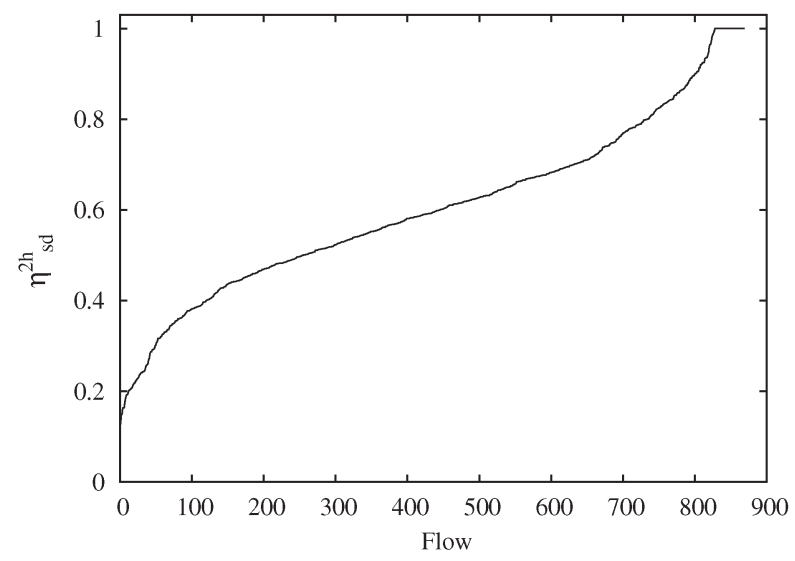

Fig. 4. Two-hop efficiencies $\eta_{s d}^{2 h}$ under the single-flow scenario for Umass buses.

\section{EXPerimental Mobile Networks}

We have analyzed an experimental vehicular mobile ad hoc network using the data obtained from the DieselNet [7] network running on Umass campus during the Spring quarter in 2005. This network consisted of 30 buses offering the campus transportation service and carrying some short-range radio devices. The publicly available traces [7] refer to the radio contacts among buses, measured in terms of data transferred through Transmission Control Protocol (TCP) connections. The traces provide a sequence of records in the form of $\left(t_{c}, s_{c}, d_{c}, b_{c}\right)$, meaning that during the $c$ th contact, which was started at time $t_{c}$, bus $s_{c}$ was able to transfer $b_{c}$ bytes of data to bus $d_{c}$. If $t_{\text {tot }}$ is the total duration of the trace, it is possible to directly evaluate the service rate between two buses $i$ and $j$ as

$$
\mu_{i j}^{S}=\frac{1}{t_{\mathrm{tot}}} \sum_{c} \mathbb{I}_{s_{c}=i} \mathbb{I}_{d_{c}=j} b_{c}
$$

by summing the data over any contact $c$ with source $i$ (i.e., $s_{c}=i$ ) and destination $j$ (i.e., $d_{c}=j$ ). Note that these records also depend on the IEEE 802.11 Medium Access Control (MAC) protocol adopted in the experimentation. Furthermore, we restricted our analysis to the first three weeks, excluding weekends and weeknights (14 working days in total). This 
guaranteed a good degree of stationarity in the intercontact times.

From the traces, one can immediately derive the corresponding contact graph. This turns out to be almost fully connected. The number of its edges is approximately $86 \%$ of a complete graph. In other words, each bus got in contact with almost all of the other buses. It is interesting to note that the resulting contact graph contains significant inhomogeneous capacities. Fig. 3 visually shows the contact graph partitioned into three subgraphs: the left subgraph contains edges with small capacities, the central subgraph (the richest subgraph) contains medium-capacity edges, whereas high-capacity edges are shown in the right subgraph. In this last subgraph, we have highlighted in bold two edges with very high capacity ( $>260$ Mbytes/14 days). Observe that even if less than $40 \%$ of the overall edges have high capacity, however, the high-capacity edges provide the main contribution to transport capability.

We compared the following routing policies.

- TH-OPT: The algorithm maximizes the aggregate transmission rate [as defined in (16)] by solving the multicommodity flow problem on the contact graph defined by (9) and (10) using standard linear programming tools.

- TH-2H-OPT: The algorithm maximizes the aggregate transmission rate like TH-OPT but with the additional constraint that only one- and two-hop routes are allowed (i.e., $f_{i j}^{s d}=0$ when both $i \neq s$ and $j \neq d$ ).

- DL-OPT: The algorithm computes the minimum-delay routes as proposed in [23]. It adopts a modified version of Dijkstra's algorithm to find paths with minimum delay. Delay is defined as the time taken by a data unit to be transferred across the sequence of relays up to reaching the destination. To find the complete set of routing paths between a source $s$ and a destination $d$, the algorithms iterate among the following phases.

1) Compute the minimum-delay $\operatorname{path}^{9} \mathcal{P}_{s d}$.

2) Compute the maximum bandwidth across $\mathcal{P}_{s d}$ : $b_{s d}=\min _{(i, j) \in \mathcal{P}_{s d}}\left\{\mu_{i j}\right\}$.

3) Allocate $b_{s d}$ across $\mathcal{P}_{s d}$ and remove all the edges corresponding to fully allocated edges. The edge capacities will be updated as $\mu_{i j}-b_{s d}$.

4) On the residual graph, start again with phase 1 until no bandwidth can no longer be allocated.

This algorithm can be extended to the multiflow scenario by just modifying the two initial steps in the following way.

1) Compute the set of minimum-delay paths $\left\{\mathcal{P}_{s d}\right\}$ for all active flows.

2) Compute the bandwidth among the paths included in $\left\{\mathcal{P}_{s d}\right\}$ according to a max-min fairness algorithm [46].

Table I provides a synoptic view of the algorithms. Note that the random two-hop policy in [13] is asymptotically optimal in terms of throughput only for infinite-size networks with

\footnotetext{
${ }^{9}$ The contact graph used by DL-OPT is slightly different from the contact graph described in Section VI, since it is a multigraph storing detailed information regarding the time and the capacity of all the contact events. See [23] for details.
}

uniform contact capacities, whereas TH-2H-OPT can be considered to be its optimal version for finite networks.

For a meaningful comparison, we considered the following two traffic scenarios.

1) Single flow: Just one source-destination pair (i.e., a pair of buses corresponds to a single traffic flow) is active in the network, and all the nodes cooperate to transfer the data of such a flow. For an exhaustive analysis, we considered all possible $n(n-1)$ flows: one for each source-destination pair. In the case of Umass trace, this corresponds to 870 single-flow cases.

2) Multiflows: Each node is both the source and the destination of exactly one traffic flow whose generation rate is set for all flows equal to $\lambda$ [i.e., $\lambda_{s d}=\lambda, \forall(s, d)$ ]. All the nodes cooperate to transfer the $n$ flows active in the network. Since there are $n$ ! ways to match sources and destinations, we only considered the following two extreme cases.

a) Minimum Direct Capacity $(m D C)$ : Node pairs are selected in such a way that the sum of the capacities associated to the direct communication links between sources and destinations is minimized. As a consequence, direct communications between sources and destinations are inefficient, and multihop transmission should be exploited to maximize the throughput.

b) Maximum Direct Capacity $(M D C)$ : Node pairs are selected to maximize the same sum as above. In this case, direct communications between a source and its destination are quite efficient, and we expect that a small number of hops will be enough for maximizing the throughput.

The performance metrics we have considered are 1) aggregate throughput, 2) per-flow throughput, and 3) delay. The delay is averaged over different routing paths, weighted with the corresponding throughput along the path.

For the single-flow scenario, Table II reports the performance achievable by the three considered routing algorithms. As expected, TH-OPT obtains the highest per-flow throughput at the cost of the largest delay. By reducing the maximum number of hops, TH-2H-OPT is able to improve the delay, but at the expense of a lower throughput. To better understand the possible inefficiencies of two-hop routing in this vehicular scenario, we evaluated the two-hop throughput efficiency $\eta_{s d}^{2 h}=\mu_{s d}^{2 h} / \hat{\mu}_{s d}$ for each single flow. Fig. 4 shows the corresponding values for all 870 flows (displayed in increasing order). The average value of $\eta_{s d}^{2 h}$ is 0.603 , suggesting that moderate throughput degradation is experienced by employing a two-hop routing strategy under most traffic scenarios, like the ones previously selected. Nevertheless, the value of $\eta^{2 h}=\min _{s d} \eta_{s d}^{2 h}$ is rather small, i.e., equal to 0.1234 . As a consequence, significant throughput degradation is experienced for the worst-case source-destination pair.

By optimizing the delay, DL-OPT obtains significantly better delays than TH-OPT and TH-2H-OPT. The corresponding throughput is smaller (as expected) than TH-OPT but larger than TH-2H-OPT. That means that the best throughput-delay tradeoff cannot be achieved by TH-2H-OPT.

Fig. 5(a)-(c) provides a more detailed view of the performance achievable under the single-flow scenario, showing the 


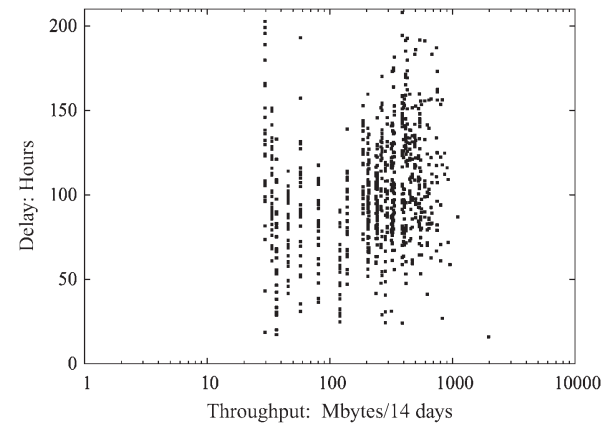

(a)

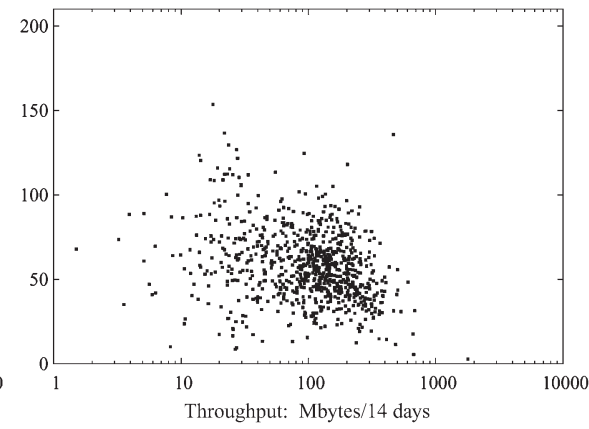

(b)

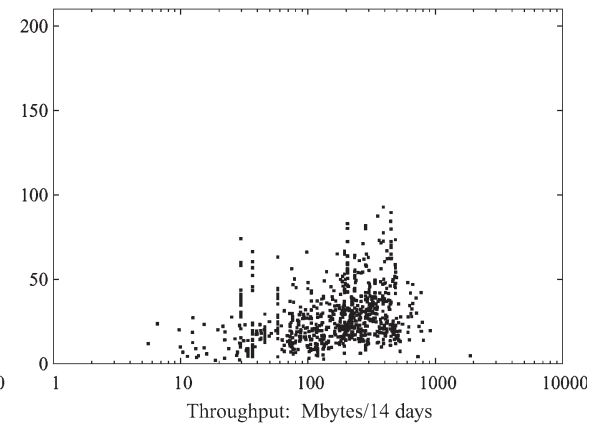

(c)

Fig. 5. Optimal throughput-delay region under single-flow scenario for Umass buses. (a) TH-OPT. (b) TH-2H-OPT. (c) DL-OPT.

TABLE III

Capacity Obtained by Optimal Routing Under the Multiflow SCENARIO FOR UMASS BUSES

\begin{tabular}{|c|c|c|c|}
\hline $\begin{array}{c}\text { Traffic } \\
\text { scenario }\end{array}$ & $\begin{array}{c}\text { Routing } \\
\text { algorithm }\end{array}$ & $\begin{array}{c}\text { Maximum total } \\
\text { capacity }\end{array}$ & $\begin{array}{c}\text { Average } \\
\text { delay }\end{array}$ \\
\hline \multirow{3}{*}{ MDC } & TH-OPT & 8.36 Gbytes/14days & 44.1 hours \\
& TH-2H-OPT & 7.02 Gbytes/14days & 22.3 hours \\
& DL-OPT & 7.31 Gbytes/14days & 13.6 hours \\
\hline \multirow{3}{*}{$m D C$} & TH-OPT & 4.09 Gbytes/14days & 109.8 hours \\
& TH-2H-OPT & 2.63 Gbytes/14days & 65.6 hours \\
& DL-OPT & 2.64 Gbytes/14days & 39.1 hours \\
\hline
\end{tabular}

optimal throughput-delay region achievable for each possible flow (represented by a point in the graph), under the three different routing policies. Fig. 5(a) shows that many groups of flows are characterized by identical throughputs but different mean overall delays. These groups share either the same source or the same destination node, where a capacity bottleneck can arise because the node sporadically gets in contact with just another node. Note that this effect particularly occurs for flows with a lower throughput. The throughput on these paths is limited by the bottleneck capacity, whereas delays depend on the specific path. Fig. 5(b) shows that TH-2H-OPT experiences better delays but at the expense of a smaller throughput. The absence of the previous phenomenon can be explained by the fact that TH-2H-OPT is not capable to saturate the bottleneck capacity, as for TH-OPT. Finally, Fig. 5(c) shows the minimum delays achievable under DL-OPT routing. We still observe groups of flows with the same throughput due to the saturation of bottleneck edges, as in TH-OPT.

For the multiflow scenario, Table III shows the aggregate performance for the two considered traffic patterns (MDC and $\mathrm{mDC}$ ). The global capacity is computed by summing the throughput of all active flows in the network. As in the singleflow case, TH-OPT achieves the best capacity, but now, TH-2HOPT and DL-OPT obtain almost the same throughput since the multiflow scenario tends to distribute the load among the nodes and $\mathrm{TH}-2 \mathrm{H}-\mathrm{OPT}$ is able to saturate all the edges as DL-OPT. The fact that DL-OPT achieves better delays indicates that there are many paths with similar capacities but different delays. DLOPT is more able than TH-2H-OPT to exploit these minimumdelay paths. In conclusion, the best throughput-delay tradeoff is not obtained by TH-2H-OPT also in the multiflow scenario.

Between the two traffic patterns, mDC highlights the throughput performance differences obtained by the three routing algorithms. This is due to the fact that several optimal paths longer than two hops are ignored by TH-2H-OPT. Such long paths are likely to also be ignored by the greedy algorithm adopted in DL-OPT since they often lead to large delays.

Figs. 6 and 7 report the detailed performance obtained by each flow under MDC and $\mathrm{mDC}$ traffic patterns. The results in these plots have been sorted in increasing order by choosing the appropriate flow sequence. Fig. 6(a) shows that TH-OPT, as expected, always outperforms the other two algorithms in terms of throughput, whereas TH-2H-OPT and DL-OPT exhibit a very similar behavior. Furthermore, because of the inhomogeneous nature of the contact graph, the throughput performance of different flows is very different (ranging over more than two orders of magnitude), even if the set of flows was chosen to maximize the direct connection capacity. Interestingly, the delays of DL-OPT are better than TH-2H-OPT, even if they are associated with the same throughput. Finally, Fig. 6(b) suggests that delays for each single flow follow the same relative performance than the aggregate delays.

Under the mDC traffic pattern, Fig. 7(a) presents the throughput achieved by the different flows. Large discrepancies are experienced in this scenario as well. In addition, the delays experienced by the flows [presented in Fig. 7(b)] are significantly different among them.

As a final comment on the numerical results discussed in this section, we note that the absolute performance indexes considered so far show that the DTN exploiting the Umass bus transport service achieves a quite poor performance in terms of both throughput and delays. This is not due to routing inefficiencies since all the considered routing schemes are optimal with respect to some performance metric and rely on centralized algorithms that exploit complete knowledge of future contacts. ${ }^{10}$ Instead, in the considered vehicular scenario, poor performance can easily be explained by considering the testbed nature of the network, exploiting (in the available traces) the mobility of only a few nodes in a large area. The nature of the transportation service itself tends to reduce the contact times between buses to minimize the delays experienced by users at bus stops.

\footnotetext{
${ }^{10}$ Notice that the absolute performance that we have computed provides an upper bound on the best performance achievable by any distributed algorithm running without full knowledge of the contacts.
} 


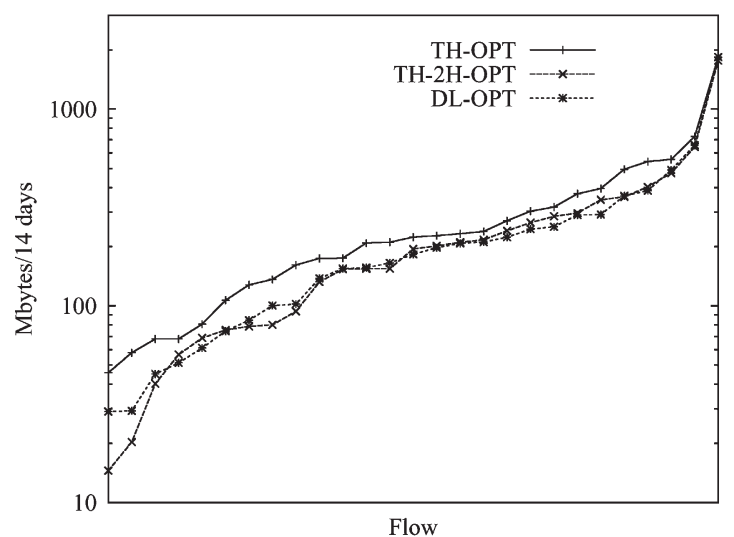

(a)

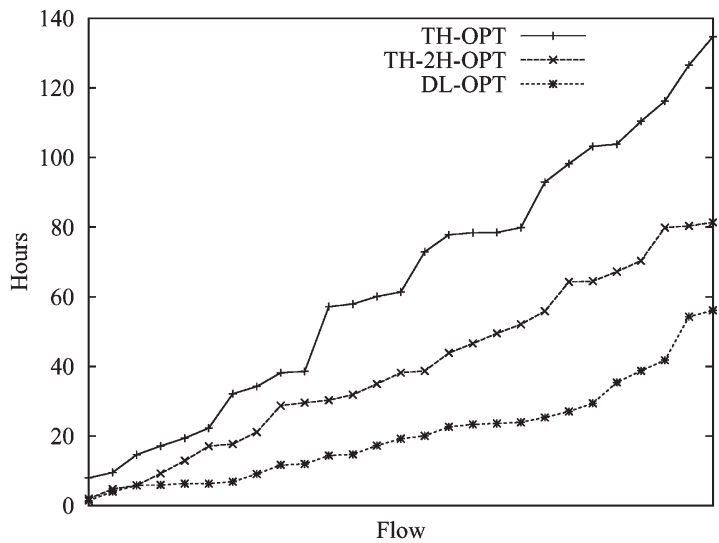

(b)

Fig. 6. Performance achievable for each flow under the MDC multiflow scenario for Umass buses. (a) Throughput. (b) Average delay.

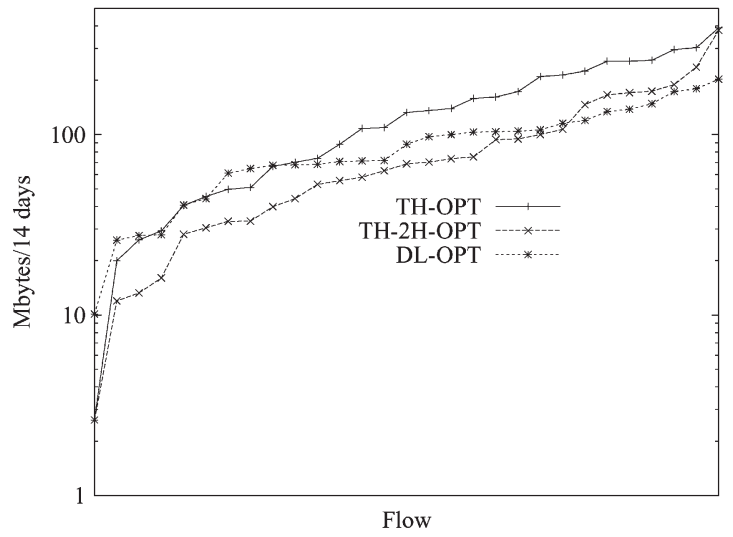

(a)

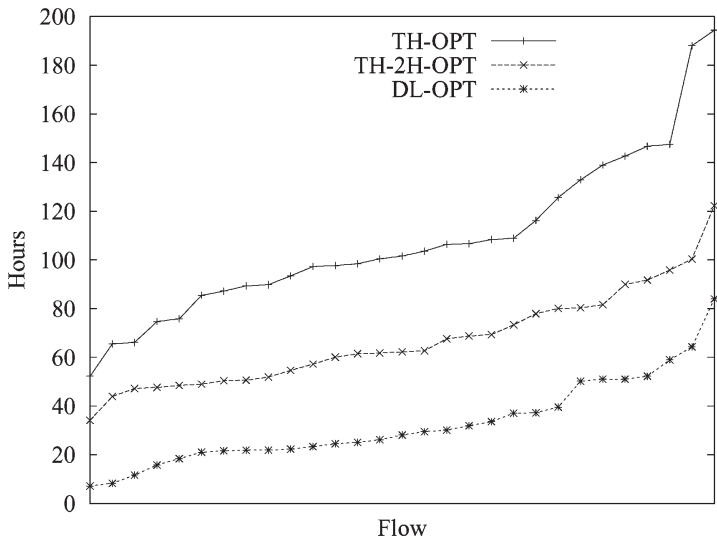

(b)

Fig. 7. Performance achievable for each flow under the mDC multiflow scenario for Umass buses. (a) Throughput. (b) Average delay.

\section{COnclusion}

In this paper, we have considered an ad hoc wireless network composed of $n$ heterogeneous mobile nodes and proposed a general methodology that allows precise characterization of its capacity region by considering the associated contact graph, highlighting several important structural properties of the system. Then, we have turned our attention to the throughput performance of the two-hop routing scheme. We have related the effectiveness of the two-hop routing strategy to the structural properties of the contact graph associated with the network. We have then presented experimental evidence that the contact times between nodes are highly inhomogeneous in a real environment. Finally, we have shown that, in realistic networks, the two-hop routing strategy result to be strongly inefficient in terms of aggregate throughput.

In a real vehicular scenario, we have assessed the capacity-delay region achievable by routing algorithms that are optimal from the throughput and delay point of views. The performance indexes that we have obtained in the considered vehicular scenario are not compatible with the qualityof-service constraints of realistic applications. However, we emphasize that our framework is general and can be applied to any vehicular network provided that the contact history is known or can easily be estimated (as in the case of scheduled mobility for public transportation systems). Our methodology provides an upper bound for the performance achievable by any routing algorithm and, for this reason, can be considered as a reference model to test the performance of future implemented routing protocols for DTNs.

\section{REFERENCES}

[1] M. Garetto, P. Giaccone, and E. Leonardi, "On the capacity of ad hoc wireless networks under general node mobility," in Proc. IEEE INFOCOM, Anchorage, AK, May 2007, pp. 357-365.

[2] M. Garetto, P. Giaccone, and E. Leonardi, "On the effectiveness of the 2-hop routing strategy in mobile ad hoc networks," in Proc. IEEE ICC, Glasgow, U.K., Jun. 2007, pp. 3108-3113.

[3] Delay Tolerant Network Research Group (DTNRG). [Online]. Available: www.dtnrg.org

[4] K. Fall, "A delay-tolerant network architecture for challenged Internets," in Proc. ACM SIGCOMM, 2003, pp. 27-34.

[5] S. Burleigh et al., "Delay-tolerant networking: An approach to interplanetary Internet," IEEE Commun. Mag., vol. 41, no. 6, pp. 128-136, Jun. 2003.

[6] A. Chaintreau, P. Hui, J. Crowcroft, C. Diot, R. Gass, and J. Scott, "Impact of human mobility on the design of opportunistic forwarding algorithms," in Proc. IEEE INFOCOM, Barcelona, Spain, Apr. 2006, pp. 1-13.

[7] J. Burgess, B. Gallagher, D. Jensen, and B. N. Levine, "MaxProp: Routing for vehicle-based disruption-tolerant networking," in Proc. IEEE INFOCOM, Barcelona, Spain, Apr. 2006, pp. 1688-1698.

[8] H. Y. Huang, P. E. Luo, M. Li, D. Li, X. Li, W. Shu, and M. Y. Wu, "Performance evaluation of SUVnet with real-time traffic data," IEEE Trans. Veh. Technol., vol. 56, no. 6, pp. 3381-3396, Nov. 2007.

[9] M. Sede, X. Li, D. Li, M. Y. Wu, M. Li, and W. Shu, "Routing in largescale buses ad hoc networks," in Proc. IEEE WCNC, Apr. 2008, pp. 27112716. 
[10] P. Juang, H. Oki, Y. Wang, M. Martonosi, L.-S. Peh, and D. Rubenstein, "Energy-efficient computing for wildlife tracking: Design tradeoffs and early experiences with ZebraNet," in Proc. ASPLOS-X, San Jose, CA, Oct. 2002, pp. 96-107.

[11] A. Seth, D. Kroeker, M. Zaharia, S. Guo, and S. Keshav, "Low-cost communication for rural Internet kiosks using mechanical backhaul," in Proc. ACM MobiCom, Los Angeles, CA, Sep. 2006, pp. 334-345.

[12] P. Gupta and P. R. Kumar, "The capacity of wireless networks," IEEE Trans. Inf. Theory, vol. 46, no. 2, pp. 388-404, Mar. 2000.

[13] M. Grossglauser and D. N. C. Tse, "Mobility increases the capacity of ad hoc wireless networks," IEEE/ACM Trans. Netw., vol. 10, no. 2, pp. 477486, Aug. 2002.

[14] S. Toumpis and A. Goldsmith, "Large wireless networks under fading, mobility, and delay constraints," in Proc. IEEE INFOCOM, Hong Kong, Mar. 2004, pp. 609-619.

[15] A. El Gamal, J. Mammen, B. Prabhakar, and D. Shah, "Throughput-delay tradeoff in wireless networks," in Proc. IEEE INFOCOM, Hong Kong, Mar. 2004, pp. 464-475.

[16] G. Sharma, R. R. Mazumdar, and N. B. Shroff, "Delay and capacity tradeoffs in mobile ad hoc networks: A global perspective," in Proc. IEEE INFOCOM, Barcelona, Spain, Apr. 2006, pp. 981-992.

[17] N. Sarafijanovic-Djukic, M. Piorkowski, and M. Grossglauser, "Island hopping: Efficient mobility-assisted forwarding in partitioned networks," in Proc. IEEE SECON, Reston, VA, Sep. 2006, pp. 226-235.

[18] W.-J. Hsu and A. Helmy, "On nodal encounter patterns in wireless LAN traces," in Proc. WiNMee, Boston, MA, 2006, pp. 1-10.

[19] J. Leguay, T. Friedman, and V. Conan, "Evaluating mobility pattern space routing for DTNs," in Proc. IEEE INFOCOM, Barcelona, Spain, Apr. 2006, pp. 1-10

[20] M. Balazinska and P. Castro, "Characterizing mobility and network usage in a corporate wireless local-area network," in Proc. ACM MobiSys, San Francisco, CA, May 2003, pp. 303-316.

[21] J. H. Kang, W. Welbourne, B. Stewart, and G. Borriello, "Extracting places from traces of locations," ACM SIGMOBILE Mobile Comput. Commun. Rev., vol. 9, no. 3, pp. 58-68, Jul. 2005.

[22] A. Boukerche, S. K. Das, and A. Fabbri, "Analysis of a randomized congestion control scheme with DSDV routing in ad hoc wireless networks," J. Parallel Distrib. Comput., vol. 61, no. 7, pp. 967-995, Jul. 2001.

[23] S. Jain, K. Fall, and R. Patra, "Routing in a delay tolerant network," in Proc. ACM SIGCOMM, 2004, pp. 145-158.

[24] W. Zhao, M. Ammar, and E. Zegura, "A message ferrying approach for data delivery in sparse mobile ad hoc networks," in Proc. ACM MobiHoc, 2004, pp. 187-198.

[25] C. Liu and J. Wu, "Scalable routing in delay tolerant networks," in Proc. ACM MobiHoc, 2007, pp. 51-60.

[26] A. Vahdat and D. Becker, "Epidemic routing for partially connected ad hoc networks," Duke Univ., Durham, NC, Tech. Rep. CS-200 006, Apr. 2000.

[27] T. Small and Z. J. Haas, "Resource and performance tradeoffs in delaytolerant wireless networks," in Proc. ACM Workshop Delay-Toler. Netw., Aug. 2005, pp. 260-267.

[28] T. Spyropoulos, K. Psounis, and C. S. Raghavendra, "Spray and wait: An efficient routing scheme for intermittently connected mobile networks," in Proc. ACM Workshop Delay-Toler. Netw., Aug. 2005, pp. 252-259.

[29] A. Lindgren, A. Doria, and O. Schelen, "Probabilistic routing in intermittently connected networks," ACM SIGMOBILE Mobile Comput. Commun. Rev., vol. 7, no. 3, pp. 19-20, Jul. 2003.

[30] H. Dubois-Ferriere, M. Grossglauser, and M. Vetterli, "Age matters: Efficient route discovery in mobile ad hoc networks using encounter ages," in Proc. ACM MobiHoc, Annapolis, MD, Jun. 2003, pp. 257-266.

[31] M. Grossglauser and M. Vetterli, "Locating mobile nodes with EASE: Learning efficient routes from encounter histories alone," IEEE/ACM Trans. Netw., vol. 14, no. 3, pp. 457-469, Jun. 2006.

[32] T. Spyropoulos, K. Psounis, and C. S. Raghavendra, "Single-copy routing in intermittently connected mobile networks," in Proc. IEEE SECON, Santa Clara, CA, Oct. 2004, pp. 235-244.

[33] S. Jain, M. Demmer, R. Patra, and K. Fall, "Using redundancy to cope with failures in a delay tolerant network," in Proc. ACM SIGCOMM, 2005, pp. 109-120.

[34] X. Zhang, G. Neglia, J. Kurose, and D. Towsley, "On the benefits of random linear coding for unicast applications in disruption tolerant networks," in Proc. IEEE NETCOD Workshop, Apr. 2006, pp. 1-7.

[35] J. Widmer and J.-Y. Le Boudec, "Network coding for efficient communication in extreme networks," in Proc. ACM Workshop Delay-Toler. Netw., Aug. 2005, pp. 284-291.
[36] E. P. C. Jones, L. Li, J. K. Schmidtke, and P. A. S. Ward, "Practical routing in delay-tolerant networks," IEEE Trans. Mobile Comput., vol. 6, no. 8, pp. 943-959, Aug. 2007.

[37] S. N. Diggavi, M. Grossglauser, and D. N. C. Tse, "Even one-dimensional mobility increases ad hoc wireless capacity," IEEE Trans. Inf. Theory, vol. 51, no. 11, pp. 3947-3954, Nov. 2005.

[38] A. Al Hanbali, A. A. Kherani, R. Groenevelt, P. Nain, and E. Altman, "Impact of mobility on the performance of relaying in ad hoc networks," in Proc. IEEE INFOCOM, Barcelona, Spain, Apr. 2006, pp. 1-12.

[39] T. Camp, J. Boleng, and V. Davies, "A survey of mobility models for ad hoc network research," Wireless Commun. Mobile Comput., vol. 2, no. 5, pp. 483-502, 2002.

[40] A. Jardosh, E. Belding-Royer, K. Almeroth, and S. Suri, "Towards realistic mobility models for mobile ad hoc networks," in Proc. ACM MobiCom, San Diego, CA, 2003, pp. 217-229.

[41] A. Boukerche, Handbook on Algorithms for Wireless Networking and Mobile Computing. Boca Raton, FL: CRC, 2005.

[42] A. Saha and D. Johnson, "Modeling mobility for vehicular ad-hoc networks," in Proc. 1st ACM Int. Workshop Veh. Ad Hoc Netw., Philadelphia, PA, 2004, pp. 91-92.

[43] L. Fratta, M. Gerla, and L. Kleinrock, "The flow deviation method: An approach to the store-and-forward communication network design," Networks, vol. 3, no. 2, pp. 97-133, 1973.

[44] M. Bramson, "Convergence to equilibrium for fluid models of FIFO queueing networks," Queueing Syst., vol. 22, pp. 5-45, 1996.

[45] B. Bollobas, Random Graphs. Cambridge, U.K.: Cambridge Univ. Press, 2001.

[46] D. Bertsekas and R. Gallager, Data Networks. Englewood Cliffs, NJ Prentice-Hall, 1987.

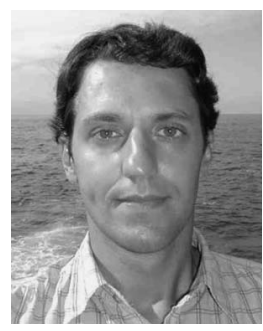

Michele Garetto (S'01-M'04) received the Dr.Ing. degree in telecommunication engineering and the $\mathrm{Ph} . \mathrm{D}$. degree in electronic and telecommunication engineering from Politecnico di Torino, Torino, Italy, in 2000 and 2004, respectively.

In 2002, he was a Visiting Scholar with the Networks Group, University of Massachusetts, Amherst. In 2004, he held a postdoctoral position with the Electrical and Computer Engineering Department, Rice University, Houston, TX. He is currently an Assistant Professor with the Università di Torino. His research interests are in the field of performance evaluation of wired and wireless communication networks.

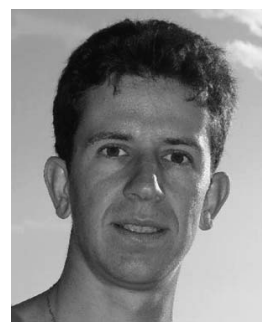

Paolo Giaccone (M'01) received the Dr.Ing. and $\mathrm{Ph} . \mathrm{D}$. degrees in telecommunications engineering from Politecnico di Torino, Torino, Italy, in 1998 and 2001, respectively.

During the summer of 1998, he was with the High Speed Networks Research Group, Lucent Technologies-Bell Labs, Holmdel, NJ. During 2000-2001, he was with the Department of Electrical Engineering, Stanford University, Stanford, CA. He is currently an Assistant Professor with the Department of Electronics, Politecnico di Torino. His main area of interest is the design of scheduling policies for high-performance routers and for wireless networks.

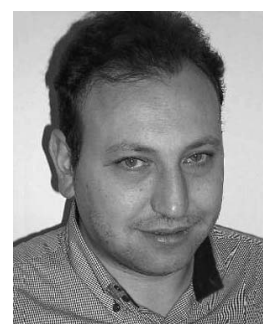

Emilio Leonardi (M'99) received the Dr.Ing. degree in electronics engineering and the Ph.D. degree in telecommunications engineering from Politecnico di Torino, Torino, Italy, in 1991 and 1995, respectively.

In 1995, he was with the Department of Computer Science, University of California at Los Angeles (UCLA). In the summer of 1999, he was with the High Speed Networks Research Group, Lucent Technologies-Bell Labs, Holmdel, NJ. In the summer of 2001, he was with the Department of Electrical Engineering, Stanford University, Stanford, CA. He is currently an Associate Professor with Politecnico di Torino. His areas of interest are all-optical networks, queueing theory, and scheduling policies for high-speed switches. 